\title{
Correction to: Creating heralded hyper-entangled photons using Rydberg atoms
}

Sutapa Ghosh, Nicholas Rivera (D), Gadi Eisenstein and Ido Kaminer (1)

Correction to: Light: Science \& Applications

https://doi.org/10.1038/s41377-021-00537-2

published online 12 May 2021

Following publication of this article ${ }^{1}$, it is noticed the Fig. 2 contained an error: three of the curves were cut. The correct Fig. 2 has been provided in this Correction.

Wavelength $(\mathrm{nm})$

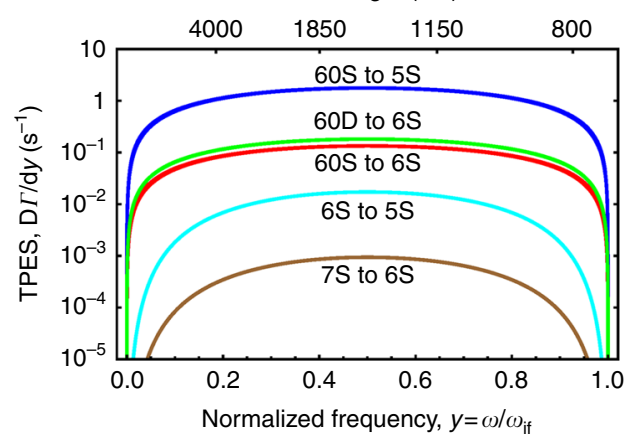

The original article has also been updated.

Published online: 21 June 2021

\section{Reference}

1. Ghosh, S., Rivera, N. \& Eisenstein, G. et al. Creating heralded hyper-entangled photons using Rydberg atoms. Light Sci. Appl. 10, 100 (2021). 